\title{
Enseñanza de redes IP con simulación en el nivel universitario: un estudio de la influencia
}

Software simulation for IP network teaching at uni-
versity level: influence study

DANIEL ARIAS FIGUEROA

Universidad Nacional de Salta

FRANCISCO JAVIER DIAZ Universidad Nacional de La Plata

MARIA CECILIA GRAMAJO Universidad Nacional de Salta

LORAINE GIMSON SARAVIA Universidad Nacional de Salta

ERNESTO SANCHEZ Universidad Nacional de Salta

ALVARO IGNACIO GAMARRA Universidad Católica de Salta

GUSTAVO DANIEL GIL Universidad Nacional de Salta

RODOLFO E. BASPINEIRO Universidad Nacional de Salta 
Resumen: Este artículo resume un estudio cuyo propósito fue determinar la influencia del uso de software de simulación en la enseñanza de conceptos y fundamentos sobre redes de computadoras, para un contexto específico. El estudio fue del tipo cuantitativo, con diseño experimental con grupo de control. Las pruebas paramétricas permitieron concluir que, existe diferencia estadísticamente significativa a favor de los estudiantes que emplearon una estrategia basada en simulación.

Palabras-claves: Enseñanza, Redes de Computadoras, Simulación, Tcp-Ip.

Abstract: This article summarizes a study whose purpose was to determine the influence of simulation software use in teaching computer networks concepts and fundamentals, for a specific context. The quantitative study was an experimental design with control group. Parametric tests led to the conclusion that there is statistically significant diference in favor of students who used a strategy based on simulation.

Keywords: Teaching, Networking, Simulation, Tcp-Ip. 


\section{Introducción}

\subsection{Marco contextual}

Este trabajo se realizó en el marco del Proyecto de Investigación No 2230/0 "Estudio de la influencia de software de simulación en la enseñanza de redes de computadoras en el nivel universitario" financiado por el Consejo de Investigaciones de la Universidad Nacional de Salta C.I.U.N.Sa. - Argentina. Los participantes del estudio fueron estudiantes de cuarto año de la carrera de Licenciatura en Análisis de Sistemas de la Universidad Nacional de Salta (UNSa) de carácter público.

El estudio se situó en la enseñanza de conceptos sobre redes IP (Internet Protocol), concretamente en el aprendizaje del protocolo DNS (Domain Name System), direccionamiento IP, ruteo IP y el protocolo ARP (Address Resolution Protocol). Estos temas se ubican en la capa de aplicación, capa de red y capa de enlace en el Modelo TCP/IP. En el nivel universitario se busca que los estudiantes puedan realizar el análisis de los distintos eventos que se producen en el funcionamiento de los protocolos estudiados.

\subsection{Antecedentes del problema}

Los conceptos y fundamentos de redes son difíciles de asimilar debido a la complejidad de los procesos involucrados que no son siempre visibles, Sakar, N. I. (2006), Goldstein, G., Leisten, S., Stark, K., Tickle, A. (2005), Javidi, G. \& Sheybani, E. (2008). Esto, sumado a los costos elevados de equipos específicos necesarios para montar un laboratorio de red, y a los escasos recursos con los que cuenta el Departamento de Informática de la Facultad de Ciencias Exactas de la Universidad Nacional de Salta (similares a los recursos con los que cuentan la mayoría de las universidades públicas en el interior del país), hacen considerar a las herramientas de simulación como una posible solución para que las prácticas sean mejor aprovechadas por los estudiantes, posibilitando además la utilización de estas herramientas fuera de los horarios de clase (Laboratorio virtual).

De acuerdo a lo dicho anteriormente, se podría resumir la problemática de la siguiente manera:

- El docente debe plantear los trabajos prácticos de laboratorio adecuándose a las características del equipo disponible, generalmente escaso (actualmente en el laboratorio de la UNSa, existen 3 enrutadores marca cisco, 5 enrutadores del tipo SOHO - Small Office Home Office y diversos conmutadores).

- La cantidad de estudiantes habitualmente es elevada (aproximadamente 20 estudiantes cada año cursan las asignaturas Redes de Computadoras I y II).

- Los equipos de hardware de red (enrutadores, conmutadores, concentradores, cableado, conectores, etc.) son costosos, y su actualización y mantenimiento también significa costos elevados, por lo que usualmente se puede contar con, a lo sumo, uno o dos dispositivos por comisión o grupos de estudiantes. Esto hace inviable los laboratorios con equipo real.

- No todos los temas se pueden abordar con una práctica sobre un entorno real.

- La curva de aprendizaje para la administración de los dispositivos en laboratorios reales es alta. Lo mismo ocurre con la conectorización física para definir una determinada topología, ya que se disponen diferentes tipos de interfaces tales como Ethernet, FastEthernet, Seriales y Puertos de Consola. Esto impide armar demasiados grupos que accedan al hardware de red.

Desde hace un tiempo, el uso de herramientas de software de simulación ha demostrado ser de gran utilidad en los procesos de enseñanza y aprendizaje en varios contextos. Concretamente, 
en redes son muchas las ventajas que se pueden enumerar; entre las que se destacan: la reducción significativa en costos de adquisición de dispositivos de red tales como enrutadores, conmutadores LAN, cableado. Así mismo, se reducen los tiempos para la puesta en marcha de los laboratorios físicos convencionales. En ese sentido, los docentes del equipo de investigación, están constantemente en la búsqueda de estrategias para mejorar la calidad de los aprendizajes. Una de esas estrategias es el uso de tecnologías de la información y la comunicación (TIC) en los procesos de enseñanza.

\subsection{Planteamiento del problema}

Esta investigación pretendió dar respuesta a la pregunta, ¿cuál es la influencia del uso de herramientas de simulación en el aprendizaje de conceptos y fundamentos sobre redes IP en estudiantes del nivel universitario? De esta pregunta principal se derivan las siguientes preguntas:

- ¿Existe diferencia estadísticamente significativa en el nivel de comprensión del concepto de los protocolos IP, entre estudiantes que reciben instrucción mediada por herramientas de simulación y estudiantes que reciben instrucción tradicional?

- ¿ ¿Existe relación entre la utilización de herramientas de simulación y la actitud de los estudiantes en el estudio de los protocolos IP?

Es importante destacar que, en el marco del proyecto de investigación mencionado, está previsto realizar experiencias similares a ésta pero con otras herramientas de simulación tales como: Kiva-NS, GNS3, MACSim entre otros, en temáticas que corresponden a las mismas asignaturas Redes de Computadoras I y II del plan de estudios de la carrera Licenciatura en Análisis de Sistemas.

Respecto de la temática para la evaluación de conceptos, el protocolo de resolución de direcciones (ARP, del inglés Address Resolution Protocol) es un protocolo de comunicaciones de la capa de red, responsable de encontrar la dirección de hardware (Ethernet MAC) que corresponde a una determinada dirección IP. Está documentado en el RFC 826. El sistema de nombres de dominio (DNS, por sus siglas en inglés, Domain Name System) es un sistema de nomenclatura jerárquico descentralizado para dispositivos conectados a redes IP como Internet o una red privada. Su función más importante es "traducir" nombres inteligibles para las personas en identificadores binarios asociados con los equipos conectados a la red. La asignación de nombres a direcciones IP es ciertamente la función más conocida de los protocolos DNS. DNS está documentado en los RFC 1034 y RFC 1035.

Quizás los aspectos más complejos de una red IP son el direccionamiento y el enrutamiento. El direccionamiento se refiere a la forma como se asigna una dirección IP y cómo se dividen y se agrupan subredes de equipos. El enrutamiento consiste en encontrar un camino que conecte una red con otra y, aunque es llevado a cabo por todos los equipos, es realizado principalmente por routers, que no son más que computadoras especializadas en recibir y enviar paquetes por diferentes interfaces de red, así como proporcionar opciones de seguridad, redundancia de caminos y eficiencia en la utilización de los recursos.

\subsection{Objetivos}

El objetivo general del estudio aquí descripto fue determinar el impacto del uso de herramientas de simulación en el aprendizaje de conceptos y fundamentos de redes de computadoras en estudiantes del nivel universitario.

A partir de este objetivo se plantearon los siguientes objetivos específicos:

- Establecer la relación entre la utilización de la simulación y la actitud de los estudiantes hacia el estudio de los protocolos IP; 
- Determinar si existe diferencia significativa en el nivel de comprensión de los principios de los protocolos mencionados, entre estudiantes que reciben instrucción mediada por herramientas de simulación y estudiantes que reciben instrucción tradicional.

\subsection{Justificación}

Este estudio aporta evidencia empírica sobre la incidencia del uso de herramientas de simulación en la enseñanza de conceptos de redes de computadoras en el nivel universitario. Existen importantes estudios sobre el tema orientados hacia otros niveles de educación y otras áreas de la ciencia tales como García y Gil (2006); Esquembre (2005); Kofman, Catalán y Concari (2004); Giorgi, Cámara y Kofman (2004); Fogliati, Catalán y Concari (2004); Casadei, Cuicas, Debel y Álvarez (2008). Asimismo, existen estudios enfocados a la física, química y matemáticas, Becerra (2005); Amaya (2008). En el área específica de la redes de computadoras se puede mencionar a Cameron, B. (2003), Zhu, S. Y. (2011).

Esta investigación beneficiará a los diferentes actores del proceso educativo: docentes, investigadores y autoridades educativas. Los resultados de esta investigación permitirán tomar importantes decisiones sobre la inclusión de herramientas TIC como medios para favorecer el aprendizaje de conceptos y fundamentos no solo de redes de computadoras.

\subsection{Limitaciones del estudio}

El estudio se desarrolló durante el período 2014-2016. En el año 2014, en el contexto de la asignatura Redes de Computadoras II. En los años 2015 y 2016, en el contexto de la asignatura Redes de Computadoras I. El planteamiento, marco teórico y metodología se repitió en todos los casos. La estrategia pedagógica definida fue el aprendizaje mediado por herramienta de simulación en una parte específica de las redes IP, para un nivel específico de enseñanza (universitario de grado).

Es importante destacar que los hallazgos de esta investigación no pueden ser generalizados a otros contextos de aprendizaje; primero, por el tamaño de la muestra seleccionada para desarrollar el estudio, y segundo, por el propio diseño de investigación realizado.

A continuación, en la Tabla 1 se presenta un cuadro con el detalle de la muestra, asignaturas, cantidad de estudiantes que formaron parte del grupo experimental y el grupo de control en cada cohorte de los talleres con simulación y la cantidad total de estudiantes involucrados en el estudio.

\begin{tabular}{|l|l|l|l|l|l|}
\hline $\begin{array}{l}\text { Experiencia en } \\
\text { Asignatura }\end{array}$ & Temática & Período & $\begin{array}{l}\text { Grupo Experi- } \\
\text { mental }\end{array}$ & $\begin{array}{l}\text { Grupo de } \\
\text { Control }\end{array}$ & $\begin{array}{l}\text { Cantidad de } \\
\text { Estudiantes }\end{array}$ \\
\hline $\begin{array}{l}\text { Redes de Compu- } \\
\text { tadoras II }\end{array}$ & Protocolo ARP & 2014 & 5 & 5 & 10 \\
\hline $\begin{array}{l}\text { Redes de Compu- } \\
\text { tadoras I }\end{array}$ & Protocolo DNS & 2015 & 5 & 16 & 21 \\
\hline $\begin{array}{l}\text { Redes de Compu- } \\
\text { tadoras I }\end{array}$ & Direccionamiento IP & 2015 & 4 & 12 & 16 \\
\hline $\begin{array}{l}\text { Redes de Compu- } \\
\text { tadoras I }\end{array}$ & Ruteo IP & 2015 & 5 & 11 & 16 \\
\hline $\begin{array}{l}\text { Redes de Compu- } \\
\text { tadoras I }\end{array}$ & Direccionamiento IP & 2016 & 6 & 6 & 12 \\
\hline $\begin{array}{l}\text { Redes de Compu- } \\
\text { tadoras I }\end{array}$ & Ruteo IP & 2016 & 5 & 8 & 13 \\
\hline $\begin{array}{c}\text { Total de estu- } \\
\text { diantes }\end{array}$ & & & $\mathbf{3 0}$ & $\mathbf{5 8}$ & $\mathbf{8 8}$ \\
\hline
\end{tabular}


Tabla 1: Estudiantes por asignatura que participaron del estudio

Para el taller con simulación se seleccionaron aleatoriamente la mitad de los estudiantes y se procedió a la resolución de una guía práctica específicamente diseñada para cada protocolo, con problemas propuestos para resolver con el simulador. Previamente, los dos grupos realizaron la práctica tradicional en el aula. A fin de que la investigación interfiriera lo menos posible con el normal desempeño de las clases, fue necesario seleccionar un diseño metodológico adecuado.

Cabe destacar también que los grupos bajo estudio pueden considerarse homogéneos o en igualdad de condiciones al inicio de cada experiencia, ya que se analizaron los planes de estudio correspondientes y el contenido de los programas de las asignaturas correlativas previas, y los mismos no contienen los conceptos evaluados en esta experiencia. Además, no se incluyeron los estudiantes recursantes en ninguno de los grupos ni tratamientos.

Respecto del método tradicional de enseñanza de redes en el Departamento de Informática, se puede argumentar que, debido a las limitaciones comentadas, no todos los temas impartidos van acompañados de una práctica con laboratorio con equipo real. En general, en las dos asignaturas involucradas a esta investigación, se realizan laboratorios integradores de conceptos con equipo real, hacia el final del cursado de las mismas.

\subsection{El software de simulación}

Existe una variedad de herramientas de simulación que permiten una fácil implementación y análisis de sistemas de redes complejos. Estos simuladores ofrecen un abanico grande de posibilidades al usuario, desde realizar una simulación con protocolos y modelos estándares, hasta tener la posibilidad de programar los suyos propios. Por ello, es importante hacer una valoración de las opciones existentes para poder elegir la herramienta más adecuada para cada caso en particular.

A continuación se presentan algunos de los simuladores de red más utilizados en la actualidad haciendo una breve descripción de cada uno.

\subsubsection{Common Open Research Emulator (CORE)}

El Laboratorio de Investigación Naval Militar de los Estados Unidos bajo el código 5520 (área de "Redes y Comunicación Subdivisión de Sistemas") apoya el proyecto Open Source "Common Open Research Emulator (CORE)", herramienta para la emulación de redes de computadoras. Esta herramienta consiste en una interfaz gráfica para crear topologías de redes virtuales, y módulos Python para la emulación de red.

Algunas de sus principales características son: es eficiente y escalable, dispone de una interfaz fácil de usar y es muy configurable. CORE se utiliza para la investigación de protocolos, demostraciones, pruebas de plataformas, evaluación de escenarios de redes, estudio de seguridad y escalabilidad de redes.

\subsubsection{Integrated Multiprotocol Network Emulator Simulator (IMUNES)}

IMUNES es un software de red integrada multiprotocolo, que emula y simula redes basadas en IP sobre el sistema operativo FreeBSD. Fue creado por un equipo de investigadores y educadores en la Universidad de Zagreb. IMUNES es una herramienta para la investigación de redes y sistemas distribuidos.

La emulación/simulación está basado en el kernel del sistema operativo FreeBSD, dividido en múltiples nodos virtuales que pueden ser interconectados a través de enlaces a nivel del núcleo para formar topologías de red complejas. Cada nodo virtual tiene su propio espacio de direcciones (IPv4 o IPv6), configuración de firewall, interfaz socket, autenticación y cifrado. Todos los nodos en IMUNES operan como si fueran nodos reales. 


\subsubsection{Graphical Network Simulator (GNS3)}

GNS3 es un simulador gráfico de red, open source, que permite la virtualización de sistemas operativos de red como Cisco, Mikrotik, Juniper, etc. en un equipo con S.O. Windows, Linux o Mac.

Con el fin de proporcionar simulaciones completas y precisas, GNS3 trabaja en conjunto con las herramientas Dynamips, VirtualBox o VMware, Qemu y Putty. También posibilita la captura de paquetes utilizando el analizador de protocolos Wireshark.

Con GNS3 se puede experimentar características o comprobar configuraciones que necesitan ser utilizadas más tarde en dispositivos reales. Para ello es necesaria la instalación del sistema operativo de red del dispositivo (ej. Router C2600), estos sistemas vienen en un archivo de imagen por cada dispositivo que por cuestiones de licencia no son proporcionados por GNS3 y deben ser adquiridos por el usuario.

Como única desventaja se puede mencionar el requerimiento elevado de recursos, principalmente de memoria RAM y procesador para evitar bloqueos del sistema. Existe la posibilidad de realizar una instalación centralizada en un servidor, lo que permite que el requerimiento para los equipos terminales sea mínimo.

\subsubsection{KivaNS}

KivaNS es una herramienta enfocada a simular el comportamiento del protocolo IP, y especialmente el tratamiento de los datagramas y el encaminamiento de los mismos por una red. También se puede estudiar el comportamiento de los protocolos auxiliares de IP como son ICMP, ARP y de redes Ethernet.

Esta herramienta se basa en la filosofía de código abierto y fue desarrollada en Java lo cual permite independencia total de la plataforma.

El objetivo principal del entorno es ayudar a diseñar y comprender el funcionamiento de redes de datos, y en especial el encaminamiento de paquetes en la arquitectura TCP/IP, sin necesidad de una infraestructura real. KivaNS también es capaz de simular distintos tipos de errores en el funcionamiento de las redes, como la pérdida de paquetes o fallos en tablas de encaminamiento. Si bien la herramienta es flexible en cuanto al diseño de la topología de red, resulta muy acotada en cuanto a los conceptos de redes que se pueden analizar.

Una característica muy interesante de esta herramienta, es la posibilidad de exportar los diseños realizados para utilizarlos en otras aplicaciones independientes o incrustarse como applets en un documento HTML, es decir, en páginas web o plataformas de e-learning.

\subsubsection{Marionnet}

Marionnet es un simulador de redes virtuales que nació en el año 2005 bajo el patrocino de la Universidad de París XIII Paris-Nord, permite a los usuarios definir, configurar y simular redes de computadoras complejas sin la necesidad de equipos físicos como routers, switches o hubs.

Esta plataforma trabaja con el sistema operativo GNU/Linux y puede simular una red compleja. El código fuente está escrito en OCaml, con sólo algunos fragmentos de lenguaje C. Es fácil y rápido de configurar, incluso con topologías complejas, teniendo la posibilidad de revertir los cambios hechos en el sistema de archivo, lo que hace a Marionnet muy flexible.

Su principal objetivo es la enseñanza de redes de computadoras en laboratorios universitarios, por lo que es usado por varias universidades de Francia y otros países.

\subsubsection{NetSim Network Simulator}


La herramienta NetSim Network Simulator está compuesta por una suite que ofrece un aprendizaje completo en el diseño, configuración y puesta en marcha de redes, que simula el hardware y el software de Cisco Systems. Fue diseñado para ayudar al usuario a aprender la estructura de comando del sistema operativo IOS de Cisco.

NetSim Network Simulator junto con el motor virtual de paquetes crea paquetes individuales que se enrutan a través de la red simulada, permitiendo a NetSim construir una tabla de enrutamiento virtual y simular un verdadero trabajo en red. También ofrece otras herramientas adicionales como CCENT Network Simulator, CCNA Network Simulator y CCNP Network Simulator. Cada uno soporta las tecnologías y las prácticas que se necesitan para la certificación de Cisco respectiva.

NetSim además incluye lecciones y laboratorios que cubren los protocolos de enrutamiento, dispositivos de Cisco, conmutación, diseño topológico entre otros. Si bien permite diseñar una red compleja y testear su funcionalidad, no permite visualizar el comportamiento de los paquetes que viajan en la red, además de tener una fuerte dependencia de los dispositivos Cisco.

\subsubsection{Net-Simulator}

NET-Simulator es una aplicación educativa, que fue creada para ayudar a los profesores y estudiantes en el aprendizaje de las redes informáticas. Los estudiantes pueden construir redes virtuales en el entorno proporcionado por NET-Simulator. Soporta varios dispositivos virtuales como router, pc, hub y switch. Estos dispositivos son controlados por medio de la interfaz de línea de comandos.

Esta herramienta tiene su propia implementación de la primera, segunda y tercera capa del Modelo OSI de la ISO. No todos los detalles de la capa física son emulados, por lo que las especificaciones eléctricas y físicas de los dispositivos no se tienen en cuenta. Se supone que los paquetes en la segunda capa se transmiten a través de un medio como Ethernet.

Así, esta plataforma permite a los usuarios estudiar los principios de funcionamiento de los dispositivos de capa 2 y 3 , tales como hubs, switches y routers y conseguir las habilidades prácticas de la configuración del enrutamiento estático.

\subsubsection{Packet Tracer}

Packet Tracer es una herramienta de simulación interactiva para aprender las operaciones de distintos dispositivos de red en las diferentes capas del Modelo OSI. Permite que el usuario construya sus propias redes y observe el comportamiento de los paquetes de datos en su recorrido por routers, switches y demás dispositivos de red. Permite crear una topología física de la red de una menera simple e intuitiva. Existen muchas opciones de interconexión, una biblioteca de dispositivos, enlaces y equipos terminales. Una vez que el estudiante completa la configuración física y lógica de la red se pueden realizar simulaciones de conectividad verificando de esta manera la correcta configuración de la red.

El software Packet Tracer fue desarrollado por la empresa de networking Cisco, y su principal objetivo fue apoyar en el entrenamiento de los alumnos para obtener las distintas certificaciones que se ofrecen desde la academia de la misma empresa. La interfaz ofrece un "Espacio de Trabajo Lógico" que tiene asociado dos modos de operación el "Tiempo Real" y el de "Simulación":

- Modo tiempo real: en este modo se realiza el diseño de la topología de la red, agregando dispositivos y configurando los mismos, incluso verificando la funcionalidad.

- Modo simulación: en este modo de operación se visualiza de manera gráfica el comportamiento de los paquetes que viajan en la red creada.

Se puede afirmar que este simulador integra una serie de herramientas que en la práctica con equipo real, suelen ser difíciles de abordar por parte del alumno además de tener curva de aprendizaje elevada. Si bien esta plataforma fue creada para los estudiantes de la Academia Cisco, su 
diseño se independiza en cierta medida del fabricante, logrando que el alumno aprenda conceptos y fundamentos generales sobre redes.

\subsubsection{La elección del Software}

Entre la gran variedad de herramientas de simulación existentes, para esta experiencia se decidió utilizar Packet Tracer por los siguientes motivos:

- Es posible analizar los conceptos y fundamentos de las capas de aplicación, transporte, red y enlace Del Modelo OSI, utilizando una sola herramienta.

- Permite simulaciones complejas a gran escala.

- Se puede ejecutar en muchas plataformas (Linux, Windows y Android) com mínimos requerimientos de hardware.

- Se pueden visualizar los modelos de simulación y verificar su funcionalidad en un ambiente virtual.

- Es posible observar y analizar el comportamiento de los paquetes en la red en todo su trayecto (al igual que en un analizador de protocolos).

- Existe una gran comunidad de usuarios, variada documentación, ejemplos y prácticas disponibles.

- Está en continuo desarrollo y, por tanto, se agregan nuevas funcionalidades con el tiempo.

- Es un software con una interfaz de usuario basada en ventanas, que ofrece facilidades para el modelado, la descripción, la configuración y la simulación de redes.

- El programa permite ver el desarrollo por capas del proceso de transmisión y recepción de paquetes de datos (Modelo de Referencia OSI de la ISO).

- El desarrollo del modelo es relativamente rápido e intuitivo.

- Es posible recrear escenarios cuya reproducción sería muy complejo en un laboratorio con equipos reales.

- Favorece el contraste de las ideas previas.

- Es posible manipular las variables del modelo, lo que favorece el aprendizaje por descubrimiento.

- Con la misma herramienta se puede abarcar los contenidos de las asignaturas Redes de Computadoras I y II del Plan de Estudios.

\subsection{Simuladores en la enseñanza}

Una de las tareas más importantes de los diseñadores de redes de comunicaciones es el estudio del desempeño de las redes de computadoras. Esto se debe a que una mala decisión de diseño puede afectar fuertemente el desempeño de la red y provocar pérdidas económicas para la empresa.

En un contexto educativo, los simuladores de redes de comunicaciones son elementos activos de aprendizaje, ya que estas herramientas facilitan el estudio y comprensión de conceptos y fundamentos teóricos. La realización de prácticas de redes de datos sobre equipos reales, tropieza con la dificultad que los laboratorios. En general, están acotados en la cantidad y variedad de equipos, por lo que hay pocas posibilidades de variar el diseño y muchas veces se debe trabajar con una sola topología existente. Además, se encuentran presentes las dificultades propias de los laboratorios, como son la coordinación con otras personas que hacen uso del mismo, el mantenimiento y la cantidad de personas que puede contener.

Si bien un simulador no puede sustituir el trabajo directo con equipos, puede proporcionar en cambio: facilidad de acceso, manejo de diversas topologías, equipos y protocolos, rapidez en el armado, trabajo con diferentes tipos de escenarios, algunos de estos escenarios pueden ser configurados erróneamente o incompletos para corregirlos y, un punto clave, la visualización gráfica. Aunque no son reales, imitan de cerca la realidad. Otro punto a favor de los simuladores de redes 
de comunicaciones son las prácticas domiciliarias, en cualquier horario y al ritmo propio de cada estudiante.

Una de las funciones clave del docente, es la de propiciar un contexto que favorezca el uso de la herramienta, generando preguntas detonantes o problemas que los estudiantes deban resolver.

Uno de los efectos más notables de la utilización de este tipo de herramientas es la adopción de un nuevo perfil docente. El profesor asume un rol de facilitador y orientador, y entiende que debe ser el alumno el protagonista del proceso, García y Gil (2006). En esa línea, se pueden mencionar las siguientes funciones del profesor en su nuevo rol:

- Proveedor de recursos

- Organizador

- Tutor

- Investigador

- Facilitador

En ese sentido, el desarrollo de actividades utilizando herramientas de simulación involucra diversos niveles de abstracción que son usados en una estrategia instruccional. Es muy importante el desarrollo de una guía práctica, que motive al estudiante a investigar lo que ocurre cuando se modifican los parámetros. El desafío también fue utilizar esta herramienta para la enseñanza de conceptos y fundamentos en carreras de sistemas, y no para la formación de técnicos específicos en redes.

\section{Metodología}

La investigación se abordó desde el enfoque cuantitativo, con un alcance correlacional y siguiendo un diseño experimental con grupo de control. Este enfoque permitió medir el efecto que tiene sobre las variables dependientes, la manipulación intencional de la variable independiente (estrategia con software de simulación). En la literatura consultada, se encontraron varios estudios afines, Amaya (2009); Casadei et al. (2008); Debel et al. (2009); Olivero y Chirinos (2007); Rodríguez, Mena y Rubio (2009); Sierra (2005), que fueron desarrollados siguiendo un diseño cuasi-experimental.

Tal como se precisó previamente, es necesario resaltar que los hallazgos de este estudio no pueden ser extrapolados a otros contextos educativos, tanto por el tipo de muestra elegida como por el propio diseño de investigación realizado. Las dos variables dependientes que se analizaron fueron:

- Actitud de los estudiantes hacia el aprendizaje de los protocolos IP,

- $\quad$ nivel de comprensión de los principios de los protocolos IP

La fiabilidad y validez de estos instrumentos fueron validadas a través de pruebas piloto donde se calcularon coeficientes de confiabilidad.

Cada cohorte del taller con simulación se desarrolló en tres etapas. La primera de ellas, la fase pre-instruccional donde se llevó a cabo la selección de la muestra y designación de los grupos y contextualización. La segunda etapa fue la fase instruccional, donde se desarrollaron las secuencias didácticas respectivas similares para los dos grupos y además para el grupo experimental con el método con simulación. Por último, se realizó la fase post-instruccional, en la cual se aplicaron las pospruebas y se realizó el análisis de datos.

Para el análisis de los datos, se aplicó estadística descriptiva y pruebas paramétricas, Avila Blas, Orlando José (2003), mediante el uso del Paquete Estadístico para las Ciencias Sociales (Statistical Package for the Social Sciences o SPSS $®$ de IBM).

Para la prueba de conceptos, el objetivo principal fue contrastar estadísticamente si ambos grupos de estudio presentaron una diferencia significativa en cuanto a alguna medida de tendencia central o de variabilidad, a los fines de poder tomar una decisión confiable sobre el beneficio o no de la aplicación de la nueva técnica de enseñanza. Es decir, se buscó determinar si el uso 
de la herramienta de simulación como complemento a las prácticas tradicionales, favorece el aprendizaje. Dado que los grupos de trabajo fueron relativamente pequeños, esto es, tienen un tamaño muestral chico $(\mathrm{N}<=10)$, para realizar las pruebas de contraste antes mencionadas en los casos que correspondiere, se utilizaron aquellas asociadas a la distribución normal o de Gauss. En los casos en que ello no fue posible, se recurrió a las técnicas contempladas en la estadística no paramétrica (ya que prescinden de la distribución de los datos).

La idea básica en el análisis estadístico de los datos fue utilizar teoría y el software mencionado, para poder completar los resultados estadísticos descriptivos ya realizados, completándolos con estadísticas que permitan decidir por ejemplo: si existe diferencia significativa entre ambas metodologías de enseñanza. Este tipo de acciones se pudo realizar mediante test de hipótesis con respecto a medida de tendencia central, como ser media, mediana, etc.; medidas de variabilidad que permiten analizar la homogeneidad entre las respuestas de los estudiantes que participaron de las diferentes pruebas.

Para cada experiencia se realizaron los test con pruebas específicas como Kolmogorov-Smirnov, coeficientes de correlación no paramétricos y gráficos específicos que permitieron ver características no siempre mostradas por los gráficos descriptivos. También se realizó la prueba de los rangos con signo de Wilcoxon que permitió contrastar la hipótesis nula de que ambos grupos puedan mantener algún tipo de relación, es decir, no fueren independientes los tratamientos de los métodos con y sin simulación.

Otras pruebas estadísticas apropiadas para complementar los resultados obtenidos fueron: calcular estadísticos descriptivos: medidas de tendencia central (mediana) y desviación estándar como medida de variabilidad.

Para el test actitudinal se optó por una escala de valoración de Likert de 5 puntos para cada una de las variables o preguntas, donde 5 hace referencia al valor máximo y 1 al valor mínimo. Esto permite medir actitudes y conocer el grado de conformidad del encuestado con las afirmaciones propuestas.

Al respecto de la escala de Likert, existen diversas opiniones sobre el número de niveles a utilizar en la misma Edmondson (2005). Normalmente, el formato más popular consiste en una escala de Likert de 5 puntos. Un estudio empírico reciente demostró que la información obtenida en escalas con 5, 7 y 10 niveles posibles de respuesta muestra las mismas características respecto a la media, varianza, asimetría y curtosis después de aplicar transformaciones simples. La escala de Likert, al ser una escala que mide actitudes, es importante que pueda aceptar que las personas tienen actitudes favorables, desfavorables o neutras, lo cual es perfectamente normal en términos de información. Debido a ello, es importante considerar siempre que una escala de actitud puede y debe estar abierta a la posibilidad de aceptar opciones de respuesta neutrales.

Para garantizar la fiabilidad del instrumento se utilizó el coeficiente Alfa de Cronbach. La medida de la fiabilidad mediante el Alfa de Cronbach asume que los ítems (medidos en escala tipo Likert) miden un mismo constructo y que están altamente correlacionados, Welch \& Comer (1988). Cuanto más cerca se encuentre el valor del alfa a 1, mayor es la consistencia interna de los ítems analizados. La fiabilidad de la escala debe obtenerse siempre con los datos de cada muestra para garantizar la medida fiable del constructo en la muestra concreta de investigación. Como criterio general, George y Mallery (2003) sugiere un valor de 7 como aceptable.

El test actitudinal indagó sobre los siguientes puntos:

- Facilidad de uso del simulador,

- Limitaciones del simulador,

- Protocolos involucrados,

- Prácticos propuestos para el uso del simulador,

- Si permite simulaciones claras y reales,

- Facilidad para el seguimiento de los eventos,

- Facilidad para experimentar con otras topologías,

- Independencia de los fabricantes para la configuración,

- Potenciador de la educación no presencial. 


\section{Resultados}

\subsection{Comprobación de la validez y fiabilidad de los instrumentos}

Los instrumentos empleados fueron revisados por expertos quienes emitieron un concepto favorable. Se comprobó la fiabilidad y la validez de cada instrumento.

\subsection{Análisis de resultados}

A continuación se describe cada una de las seis experiencias realizadas para los temas correspondientes a los protocolos ARP, DNS, Direccionamiento IP y Ruteo IP, en las diferentes cohortes de las asignaturas involucradas. Al final de cada análisis estadístico de los datos, se presenta para cada caso un gráfico descriptivo, donde se puede apreciar el porcentaje de estudiantes que aprobaron tanto para el grupo experimental como para el grupo de control, como así también un detalle de las notas alcanzadas con la finalidad de contrastar la diferencia significativa a favor del grupo experimental en la mayoría de los casos.

La modelización de los datos se realizó con la variable taller que puede tomar dos valores, 0 para representar los alumnos que no realizaron el taller con herramientas de simulación, y 1 para aquellos alumnos que si realizaron el taller. La variable nota, refiere a la nota obtenida en la evaluación de conceptos en una escala de 0 a 100, siendo 50 el mínimo valor para la aprobación del mismo.

\subsubsection{Experiencia 1 - Protocolo ARP}

Para el test de Kolmogorov-Smirnov realizado, para la variable taller, con un nivel de significancia del $1 \%$ se acepta la hipótesis nula. Se hace notar que el software SPSS trabaja con un nivel de significancia del 5\%. Para la variable nota, con un nivel de significancia del 0,98\%, se acepta la hipótesis nula de que los datos provienen de una población con distribución normal.

Para la prueba de los rangos con signo de Wilcoxon, el valor obtenido para la significancia asintótica bilateral es aproximadamente 0,07, el cual resulta ser menor que el nivel de significancia $(0,05)$. Esto nos habilita a rechazar la hipótesis nula planteada.

El valor de la media para el grupo de experimental (estudiantes que realizaron simulación) es 75, mayor que el correspondiente a los estudiantes que no realizaron simulación 55. Se destaca además que el desvío estándar en el primer grupo es menor que el segundo grupo, lo cual significa que los estudiantes que realizaron simulación, presentan una mayor homogeneidad o menor variabilidad. Esto puede considerarse como un indicador que resalta la bondad de la enseñanza con simulación.

Del análisis realizado se puede observar en el gráfico que el $40 \%$ de los estudiantes que no realizaron el taller obtuvieron notas menores a 50 puntos, es decir, desaprobaron la evaluación. Además se pudo advertir, que los estudiantes que sí realizaron el taller, aprobaron con mejores notas, lo que se traduce en respuestas mucho más acabadas y precisas del concepto evaluado. 


\section{Resultados - Experiencia 1 ARP - 2014}

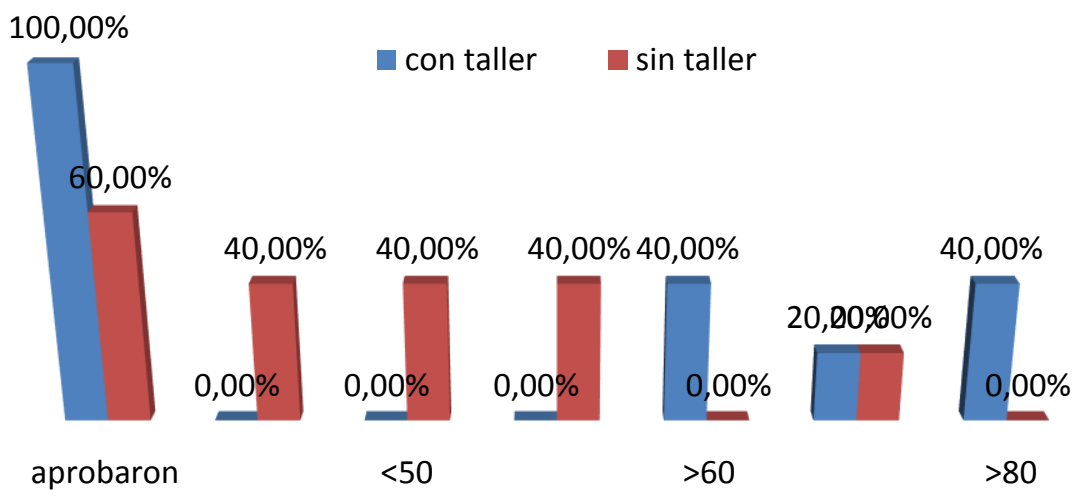

Figura 2: Redes de Computadoras II - Protocolo ARP - Año 2014

Para esta experiencia realizada, se concluye que existe diferencia estadísticamente significativa a favor de los estudiantes que emplearon una estrategia basada en simulación para el concepto del protocolo ARP evaluado.

\subsubsection{Experiencia 2 - Protocolo DNS}

Realizado el test de Kolmogorov-Smirnov, para la variable taller, con un nivel de significancia del $0,7 \%$ no se acepta la hipótesis nula. No se cuenta con elementos estadísticos suficientes para concluir que se deba rechazar la hipótesis nula, es decir, no se está en condiciones de aceptarla. Esto amerita a buscar otras alternativas de contraste de hipótesis. Existe asimetría en la variable taller, lo cual, junto con la decisión de no aceptar la hipótesis nula, lleva a tener que analizar el comportamiento de las medianas de cada grupo. Esto se hace calculando las respectivas medianas con estadística descriptiva, lo que da como resultado que el grupo experimental tiene un valor mediano muy superior al correspondiente al grupo de control. Como complemento, es aconsejable comparar estas medidas desde el punto de vista de variabilidad. Esto se hace trabajando con la desviación mediana. Para la variable nota, con un nivel de significancia del 3,89\%, se acepta la hipótesis nula de que los datos provienen de una población con distribución normal.

Para la prueba de rangos con signo de Wilcoxon, se observa que la significancia asintótica bilateral es aproximadamente 0,1 , el cual resulta NO ser menor que el nivel de significancia $(0,05)$, sin embargo el percentil $Z$ en módulo 1.633 es menor que el $Z c$ crítico de la normal al $5 \%$ que es de 1.96. Esto nos habilita a rechazar la hipótesis nula planteada.

El valor de la media para el grupo de estudiantes que realizaron simulación es 87,50 , mayor que el correspondiente a los estudiantes que no realizaron simulación 66,64 . Se destaca, además, que el desvío estándar en el primer grupo es aproximadamente del orden de la mitad del segundo grupo, lo cual significa que los estudiantes que realizaron simulación presentan una manifiesta homogeneidad o menor variabilidad. Esto puede considerarse como un indicador que resalta la bondad de la enseñanza con simulación.

Del análisis realizado se puede observar que en el grupo que realizó simulación aprobó el $100 \%$, mientras que en el grupo de control aprobó el $87 \%$. Se puede concluir también que, el grupo que realizó el taller con simulación, obtuvo notas más altas y, en el caso de la pregunta abierta, permitió inferir que se lograron respuestas mucho más acabadas y precisas del concepto evaluado. 


\section{Resultados - Experiencia 2 DNS - 2015}

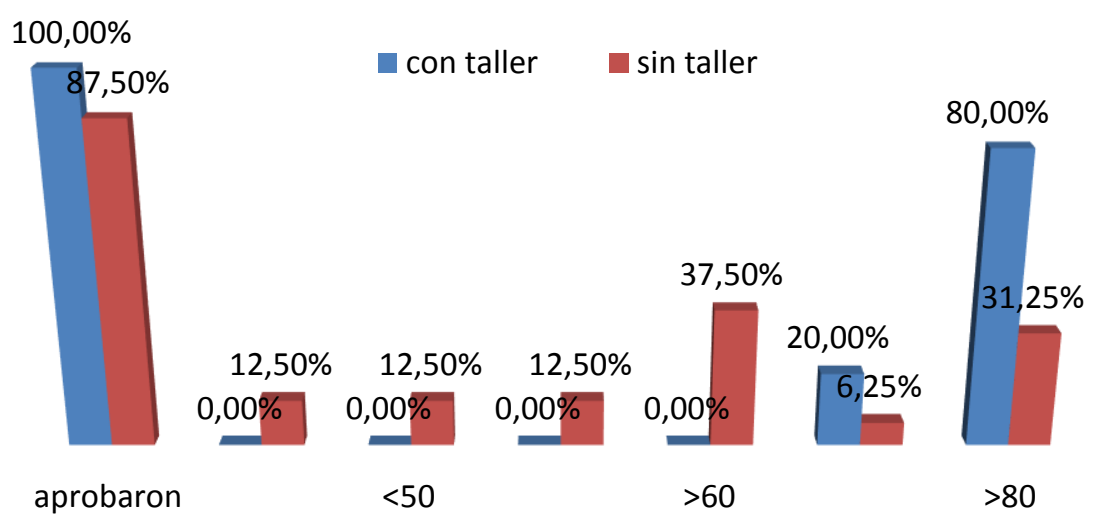

Figura 3: Redes de Computadoras I - Protocolo DNS - Año 2015

De manera que, para esta experiencia realizada, se concluye que existe diferencia estadísticamente significativa a favor de los estudiantes que emplearon una estrategia basada en simulación para el concepto del protocolo DNS evaluado.

\subsubsection{Experiencia 3 - Direccionamiento IP}

Realizado el test de Kolmogorov-Smirnov, para la variable taller, con un nivel de significancia del $0,77 \%$ se acepta la hipótesis nula. Para la variable nota, con un nivel de significancia del $0,37 \%$, se acepta la hipótesis nula de que los datos provienen de una población con distribución normal.

Para la prueba de los rangos con signo de Wilcoxon, observa que la significancia asintótica bilateral es aproximadamente 0,465 , el cual resulta NO ser menor que el nivel de significancia $(0,05)$, sin embargo el percentil $Z$ asociado $Z=-0,730$ es menor en valor absoluto que el valor crítico de la normal estándar $Z c=1,96$ con lo cual existen elementos estadísticos que permiten aceptar la hipótesis nula de independencia entre ambos métodos.

El valor de la media para el grupo de estudiantes que realizaron simulación es 64,58 , mayor que el correspondiente a los estudiantes que no realizaron simulación 45,83 . Sin embargo, se observa en este caso que el desvío estándar en el primer grupo, es levemente mayor que el del segundo grupo. Dado que las medias son distintas, a fin de comparar cual de los métodos es más eficiente relativamente, corresponde calcular el coeficiente de variación relativa (cociente entre el desvío estándar y la media). El método con menor variación relativa es el más eficiente.

El coeficiente para $G x$ es 0.339 , coeficiente para Gc es 0.413 . Coeficiente de $G x<$ Coeficiente de Gc.

Del análisis realizado se puede observar en el gráfico que el $66 \%$ de los estudiantes que no realizaron el taller obtuvieron notas menores a 50 puntos (desaprobaron). Además, se pudo advertir, que los estudiantes que sí realizaron el taller, aprobaron con mejores notas. 


\section{Resultados - Experiencia 3 Dir. IP - 2015}

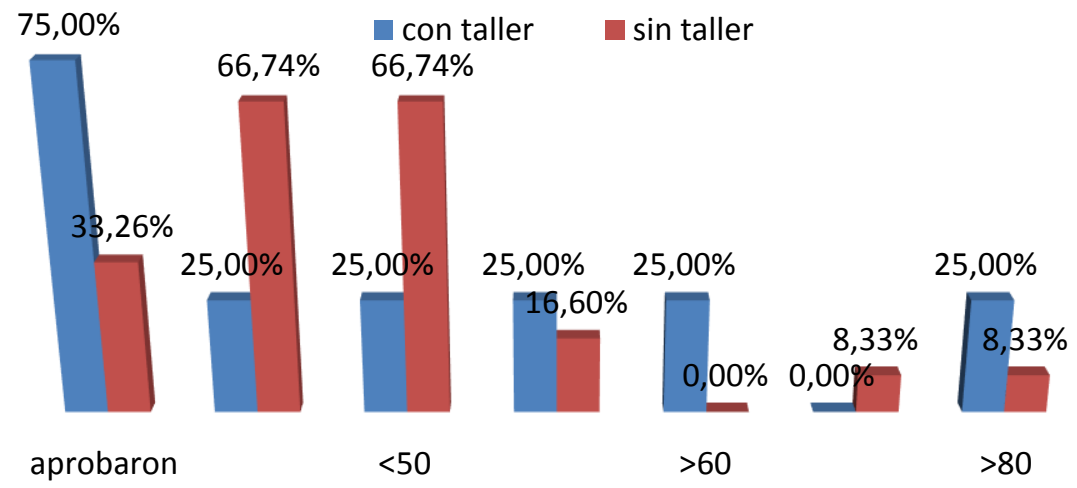

Figura 4: Redes de Computadoras I - Direccionamiento IP - Año 2015

Para esta experiencia realizada, se concluye que existe diferencia estadísticamente significativa a favor de los estudiantes que emplearon una estrategia basada en simulación para el concepto de Direccionamiento IP evaluado.

\subsubsection{Experiencia 4 - Ruteo IP}

Realizado el test de Kolmogorov-Smirnov, para la variable taller, con un nivel de significancia del $0,21 \%$ se acepta la hipótesis nula. Para la variable nota, con un nivel de significancia del $0,62 \%$, se acepta la hipótesis nula de que los datos provienen de una población con distribución normal.

Para la prueba de los rangos con signo de Wilcoxon, se observa que la significancia asintótica bilateral es aproximadamente 0,17 , el cual resulta NO ser menor que el nivel de significancia $(0,05)$, sin embargo el percentil $Z$ asociado $Z=-1,361$ es menor en valor absoluto que el valor crítico de la normal estándar $Z c=1,96$, con lo cual existen elementos estadísticos que permiten aceptar la hipótesis nula de independencia entre ambos métodos.

El valor de la media para el grupo de estudiantes que realizaron simulación es 73,33 , mayor que el correspondiente a los estudiantes que no realizaron simulación 63,64 . Sin embargo, se observa en este caso que el desvío estándar en el primer grupo, es mayor que el del segundo grupo. Dado que las medias son distintas, a fin de comparar cual de los métodos es más eficiente relativamente, corresponde calcular el coeficiente de variación relativa (cociente entre el desvío estándar y la media). El método con menor variación relativa es el más eficiente.

El coeficiente para $G x$ es 0.186 , coeficiente para Gc es 0.13 . En este caso el Coeficiente de $G x>$ Coeficiente de Gc.

Se puede observar en el gráfico que el porcentaje de aprobados-reprobados es casi el mismo para ambos grupos. Sin embargo, se puede constatar que los alumnos que realizaron el taller con simulación, obtuvieron mejores notas. 


\section{Resultados - Experiencia 4 Ruteo IP- 2015}

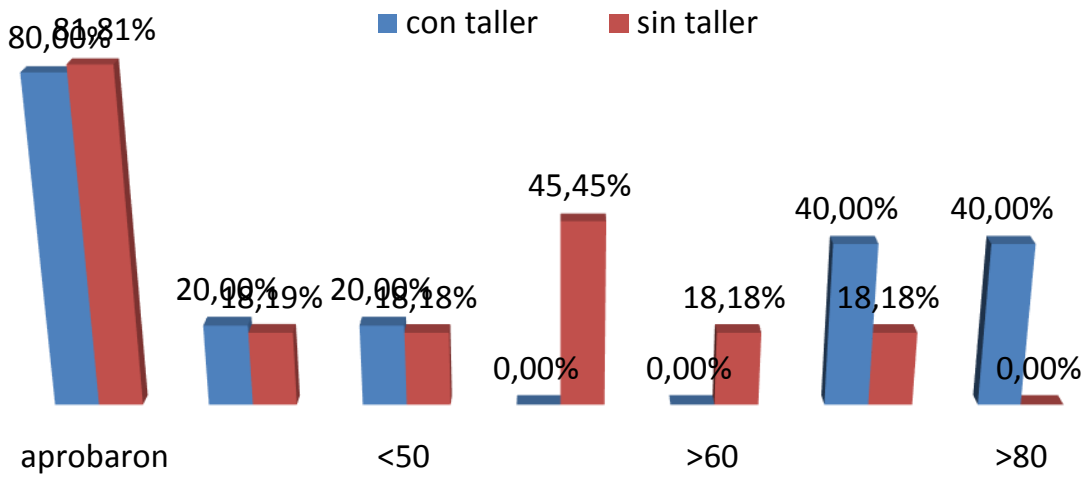

Figura 5: Redes de Computadoras I - Ruteo IP - Año 2015

Para esta experiencia realizada, se concluye que NO existe diferencia estadísticamente significativa a favor de ninguna de las estrategias, para el concepto de Ruteo IP evaluado.

\subsubsection{Experiencia 5 - Direccionamiento IP}

Realizado el test de Kolmogorov-Smirnov, para la variable taller, con un nivel de significancia del $1 \%$ se acepta la hipótesis nula. Para la variable nota, con un nivel de significancia del $0,96 \%$, se acepta la hipótesis nula de que los datos provienen de una población con distribución normal.

Para la prueba de los rangos con signo de Wilcoxon, se observa que la significancia asintótica bilateral es aproximadamente 0,02 , el cual resulta ser menor que el nivel de significancia $(0,05)$. Esto habilita a rechazar la hipótesis nula planteada.

El valor de la media para el grupo de estudiantes que realizaron simulación es 62,50 , mayor que el correspondiente a los estudiantes que no realizaron simulación 44,44 . Se destaca además que, el desvío estándar en el primer grupo es menor que el del segundo grupo, lo cual significa que los estudiantes que realizaron simulación podrían presentar mayor homogeneidad o poca variabilidad. Esto puede considerarse como un indicador que resalta la bondad de la enseñanza con simulación para esta experiencia.

Del análisis realizado se puede observar en el gráfico que el $50 \%$ de los estudiantes que no realizaron el taller obtuvieron notas menores a 50 puntos. Además se pudo advertir, que los estudiantes que si realizaron el taller, aprobaron con mejores notas, lo que se traduce en respuestas mucho más acabadas y precisas del concepto evaluado. 


\section{Resultados - Experiencia 5 Dir. IP - 2016}

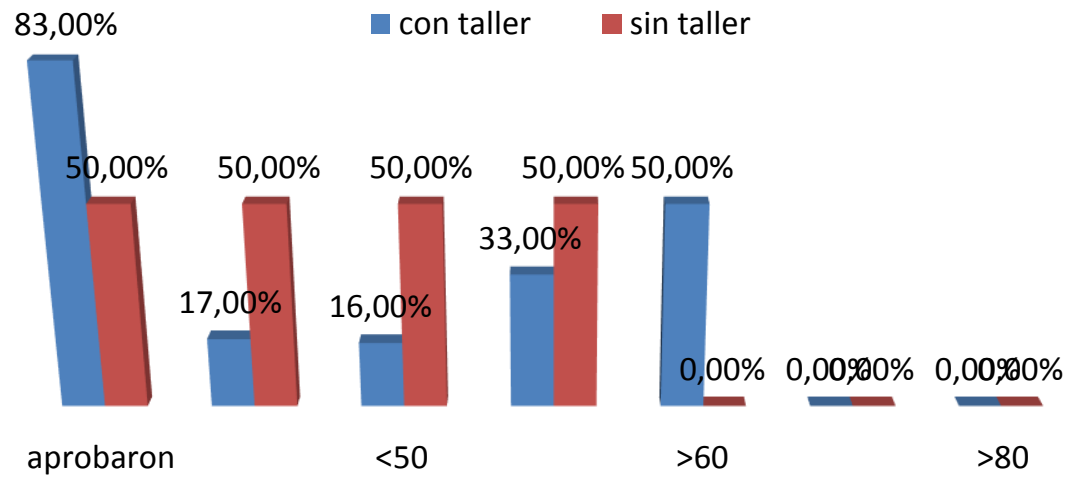

Figura 6: Redes de Computadoras I - Direccionamiento IP - Año 2016

De manera que, para esta experiencia realizada, se concluye que existe diferencia estadísticamente significativa a favor de los estudiantes que emplearon una estrategia basada en simulación para el concepto del Direccionamiento IP evaluado.

\subsubsection{Experiencia 6 - Ruteo IP.}

Realizado el test de Kolmogorov-Smirnov, para la variable taller, con un nivel de significancia del $0,58 \%$ se acepta la hipótesis nula. Para la variable nota, con un nivel de significancia del $0,92 \%$, se acepta la hipótesis nula de que los datos provienen de una población con distribución normal.

Para la prueba de los rangos con signo de Wilcoxon, se observa que la significancia asintótica bilateral es aproximadamente 0,465 , el cual resulta no ser menor que el nivel de significancia $(0,05)$, sin embargo el percentil $Z$ asociado $Z=-0,730$ es menor en valor absoluto que el valor crítico de la normal estándar $Z c=1,96$ con lo cual existen elementos estadísticos que permiten aceptar la hipótesis nula de independencia entre ambos métodos.

El valor de la media para el grupo de estudiantes que realizaron simulación es 60,00, mayor que el correspondiente a los estudiantes que no realizaron simulación 51,04. Sin embargo, se observa en este caso que el desvío estándar en el primer grupo, es levemente mayor que el del segundo grupo. Dado que las medias son distintas, a fin de comparar cual de los métodos es más eficiente relativamente, corresponde calcular el coeficiente de variación relativa (cociente entre el desvío estándar y la media). El método con menor variación relativa es el más eficiente.

El coeficiente para $G x$ es 0.228 , coeficiente para Gc es 0.253 . Coeficiente de $G x<$ Coeficiente de $G c$.

Del análisis realizado se puede observar en el gráfico que el $25 \%$ de los estudiantes que no realizaron el taller, obtuvieron notas menores a 50 puntos (reprobaron), mientras que los que si realizaron el taller, el $40 \%$ obtuvo notas menores a 60 puntos. Además, se pudo advertir que los estudiantes que realizaron el taller aprobaron con mejores notas, hay un $20 \%$ con notas mayores que 70 puntos. 


\section{Resultados - Experiencia 6 Ruteo IP - 2016}

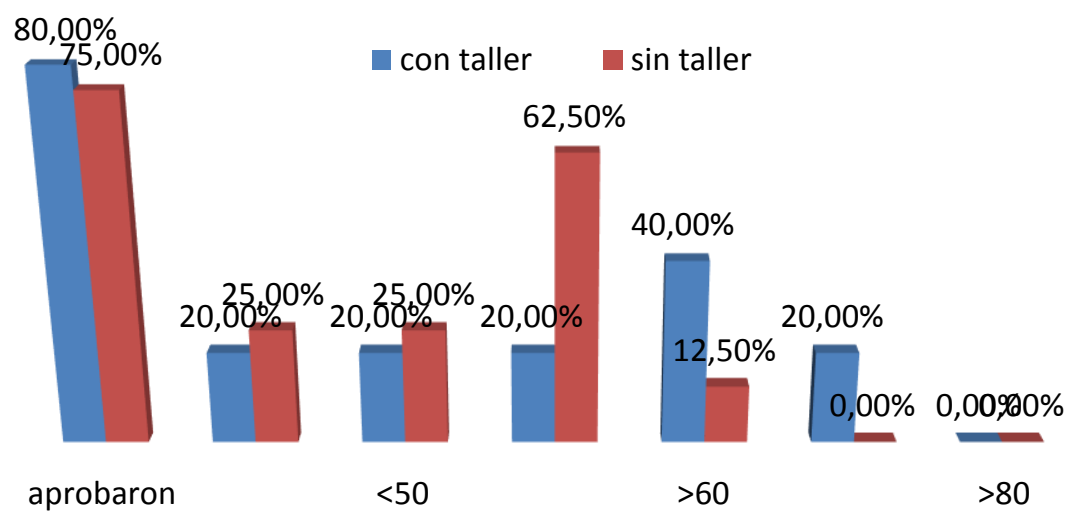

Figura 7: Redes de Computadoras I - Ruteo IP - Año 2016

De manera que, para esta experiencia realizada, se concluye que existe diferencia estadísticamente significativa a favor de los estudiantes que emplearon una estrategia basada en simulación para el concepto de Ruteo IP evaluado.

\section{Conclusiones}

\subsection{Hallazgos}

Los resultados obtenidos en las experiencias descriptas previamente, donde se trataron temas correspondientes a las Capa de Aplicación, Capa de Transporte y Capa de Red del Modelo TCP/IP, evidencian que los estudiantes consideran por una parte que la herramienta utilizada cuenta con toda la potencialidad para realizar simulaciones claras de los protocolos estudiados y por otro lado consideran apropiados los laboratorios propuestos. De manera que, en relación con el primer objetivo del estudio, se establece que, para el nivel universitario, la utilización del software de simulación tiene una incidencia significativamente alta en el desarrollo de una buena actitud hacia el estudio de las asignaturas Redes de Computadoras I y II del plan de estudios de la Licenciatura en Análisis de Sistemas.

En relación al segundo objetivo del estudio, y en función de los resultados obtenidos en las experiencias descriptas anteriormente, donde se realizaron evaluaciones de los temas Protocolo ARP, Protocolo DNS, Direccionamiento IP y Ruteo IP, se evidencia que el uso de software de simulación mejora significativamente el nivel de comprensión de los conceptos evaluados.

Por lo expuesto, se concluye que existe diferencia estadísticamente significativa a favor de los estudiantes que emplearon una estrategia basada en simulación en el nivel universitario.

\subsection{Recomendaciones}

Como futuras investigaciones se sugieren estudios sobre el impacto de software de simulación en otros ámbitos de las redes, así como en otras asignaturas del plan de estudios de la carrera. Asimismo, es importante valorar la incidencia de esta herramienta en otros niveles educativos. Se recomiendan estudios cualitativos que permitan conocer con mayor profundidad el nivel de conceptualización alcanzado por los estudiantes con el uso del software de simulación y ampliar la visión sobre el tema. Finalmente, otro punto a considerar es la influencia de diversos factores en la eficacia del software.

En cuanto a las debilidades del estudio, se debe tener en cuenta el tamaño pequeño de la muestra, por lo que las conclusiones de la investigación no pueden ser transferidas a otros contextos. Otro factor que influye en el estudio es la homogeneidad en el nivel de habilidad para 
utilizar este tipo de herramientas, la formación en informática de los alumnos facilita las experiencias. Por otro lado, la rigidez del enfoque cuantitativo limita la comprensión del fenómeno.

\section{Referencias}

ARIAS FIGUEROA, D. (2015). Redes de Computadoras I con Packet Tracer, Editorial de la Universidad Nacional de Salta - Argentina, EUNSa. ISBN 978-987-633-132-6-1; 1a ed. Salta - EBook - CDD 004.68.

ARIAS FIGUEROA, D., GIL, G., GIMSON, L. (2016). Estudio de la influencia del uso del simulador KIVA-NS en la enseñanza de redes IP. Décima Quinta Conferencia Iberoamericana en Sistemas, Cibernética e Informática (CISCI 2016) Orlando, Florida, EE.UU.

ARIAS FIGUEROA, D. \& SANCHEZ, E. (2018). Redes de Computadoras II con Packet Tracer, Editorial de la Universidad Nacional de Salta - Argentina, EUNSa. ISBN 978-987-633-527-0; 1a ed. Salta - E-Book - CDD 004.678.

AVILA BLAS, Orlando José (2003). Probabilidad y estadística inferencial: teoría y aplicaciones. ISBN: 978-987-9381-23-6. Editorial: Universidad Nacional de Salta.

CAMERON, B. (2003). Effectiveness of simulation in a hybrid online networking course. Quarterly Review of Distance Education, 4(1), 51.

DIXON, M. W., MCGILL, T. J. \& KARISOON, J. M (1997). Using a Network Simulation Package to Teach the Client-server Model. Proceedings of the 2 nd Conference on Integrating Technology into Computer Science Education, pp. 71-73.

GATTO, D. (1993). The use of interactive computer simulations in training. Australian Journal of Educational Technology, 9(2), 144-156.

GOldSTEIN, G., LEISTEN, S., STARK, K., TICKLE, A. (2005). Using a Network Simulation Tool to Engage Students in Active Learning Enhances Their Understanding of Complex Data Communications Concepts, Proceedings of the 7th Australasian conference on Computing Education, pp. 223-228.

JAVIDI, G. \& SHEYBANI, E. (2008). Content-Based Computer Simulation of a Networking Course: An Assessment, Journal of Computers, Vol. 3, No. 3, pp. 64-72.

KUROSE, J. F. \& ROSS, K. W. (2015). Computer Networking: A Top-Down Approach. 6th Edition. Pearson Education. ISBN: 9780132856201.

SAKAR, N. I. (2006). Teaching TCP/IP Networking Using Practical Laboratory Exercises, International Journal of Information and Communication Technology Education, Vol. 2, No. 4, pp. 3950.

Simulador Common Open Research Emulator (CORE). Enlace https://www.nrl.navy.mil/itd/ncs/products/core

Simulador GNS3. Enlace http://www.gns3.net

Simulador Integrated Multiprotocol Network Emulator Simulator (IMUNES). Enlace http://imunes.net/

Simulador Kiva-NS. Enlace http://www.disclab.ua.es/kiva/

Simulador Marionnet. Enlace https://www.marionnet.org/site/index.php/en/

Simulador Net-Simulator. Enlace http://www.net-simulator.org/en/index.shtml 
Simulador Packet Tracer. Enlace http://www.cisco.com/web/AR/index.html

YAVERBAUM, G., \& NADARAJAN, U. (1996). Learning basic concepts of telecommunications: an experiment in multimedia and learning. Computers \& Education, 26(4), 215-224.

ZHU, S. Y. (2011). Teaching Computer Networks through Network Simulation Programs. Faculty of Business, Computing and Law - School of Computing. University of Derby. Learning Teaching \& Assessment Conference.

Recebido em novembro de 2016

Aprovado para publicação em julho de 2018

\section{Daniel Arias Figueroa}

Centro de Investigación y Desarrollo en Informática Aplicada - C.I.D.I.A., Facultad de Ciencias Exactas de La Universidad Nacional de Salta, Salta, Provincia de Salta, Argentina. E-mail: daaf@cidia.unsa.edu.ar

\section{Francisco Javier Díaz}

Facultad de Informática de la Universidad Nacional de La Plata, Ciudad de La Plata, Provincia de Buenos Aires, Argentina. Email: jdiaz@unlp.edu.ar

María Cecilia Gramajo

Facultad de Ciencias Exactas de la Universidad Nacional de Salta, Salta, Provincia de Salta, Argentina. E-mail: cgramajo@unsa.edu.ar

\section{Loraine Gimson Saravia}

Centro Centro de Investigación y Desarrollo en Informática Aplicada - C.I.D.I.A., Facultad de Ciencias Exactas de La Universidad Nacional de Salta, Salta, Provincia de Salta, Argentina. E-mail: loraine@cidia.unsa.edu.ar

\section{Ernesto Sánchez}

Centro de Investigación y Desarrollo en Informática Aplicada - C.I.D.I.A., Facultad de Ciencias Exactas de La Universidad Nacional de Salta, Salta, Provincia de Salta, Argentina. E-mail: esnachez@cidia.unsa.edu.ar

\section{Álvaro Ignacio Gamarra}

Centro de Investigación y Desarrollo en Informática Aplicada - C.I.D.I.A., Facultad de Ciencias Exactas de La Universidad Nacional de Salta, Salta, Provincia de Salta, Argentina. E-mail: alvaroig@cidia.unsa.edu.ar

\section{Gustavo Daniel Gil}

Centro de Investigación y Desarrollo en Informática Aplicada - C.I.D.I.A., Facultad de Ciencias Exactas de La Universidad Nacional de Salta, Salta, Provincia de Salta, Argentina. E-mail: gdgil@unsa.edu.ar

\section{Rodolfo E. Baspineiro}

Centro de Investigación y Desarrollo en Informática Aplicada - C.I.D.I.A., Facultad de Ciencias Exactas de La Universidad Nacional de Salta, Salta, Provincia de Salta, Argentina. E-mail: rbaspineiro@cidia.unsa.edu.ar 